\title{
Erratum: Asymptotic Behavior of Periodic Dynamical on Banach Spaces $(*)$.
}

\author{
Marshall Sremrod (Jerusalem, Israel) (**)
}

There are errors in the proofs of Lemma 2.3 and Theorem 3.1. These are due to a mistake in Definition 2.7. The following revisions should be made:

1) After the words $« p \in \gamma^{+}\left(t_{0}, \varphi\right) »$ in Definition 2.7 insert «such that for each $\alpha$ there exists a sequence of integers $\left\{k_{j}\right\}$, increasing $k_{j} \rightarrow \infty$, so that $u\left(\sigma+k_{j} T, t_{0}, \varphi\right) \rightarrow U_{\alpha}\left(\sigma, t_{0}, p\right)$ uniformly for $\sigma$ in compact subsets of $R »$.

2) Witb the revision given 1) the inclusion $\mathrm{I}^{+}\left(t_{0}, \varphi\right) \subset L^{+}\left(t_{0}, \varphi\right)$ in the proof of Lemma 2.3 is now valid. This was not necessarily true as originally given.

3) The proof of Theorem 3.1 should now read as follows:

Since $G$ is compact and $V$ is periodic in $t, V\left(t, u\left(t, t_{0}, \varphi\right)\right)$ is nonincreasing and bounded from below for $t \geq t_{0}$. Hence, $\lim _{t \rightarrow \infty} V\left(t, u\left(t, t_{0}, \varphi\right)\right)=c$, a constant. Also, since $G$ is compact, $\gamma+\left(t_{0}, \varphi\right)$ is non-empty and by Lemma $2.2 \Gamma+\left(t_{0}, \varphi\right)$ is non-empty. Lèt $\bigcup_{\sigma \in R} U_{\alpha}\left(\sigma, t_{0}, p\right)$ be an arbitrary element of $\mathrm{I}+\left(t_{0}, \varphi\right)$ for some $p \in \gamma+\left(t_{0}, \varphi\right), \alpha \in \Lambda$. For each $\sigma \in R$ we have $u\left(\sigma+k_{j} T, t_{0}, \varphi\right) \rightarrow$ $\rightarrow U_{x}\left(\sigma, t_{0}, p\right)$ uniformly for $\sigma$ in compact subsets of $R$ for some inoreasing sequence of integers $\left\{k_{j}\right\}, k_{j} \rightarrow \infty$. So for each $\sigma \in R$ we have $V\left(\sigma, u\left(\sigma+k_{j} T\right.\right.$, $\left.\left.t_{0}, \varphi\right)\right) \rightarrow V\left(\sigma, U_{x}\left(t, t_{0}, p\right)\right)$ as $j \rightarrow \infty$ and by periodicity of $V, V\left(\sigma+k_{j} T, u(\sigma+\right.$ $\left.\left.+k_{j} T, t_{0}, p\right)\right) \rightarrow V\left(\sigma, D_{\alpha}\left(\sigma, t_{0}, p\right)\right)$ as $j \rightarrow \infty$. Hence it follows that for all $\sigma \in R, V\left(\sigma, U_{\alpha}\left(\sigma, t_{0}, p\right)\right)=c$ and $\dot{V}\left(\sigma, U_{\alpha}\left(\sigma, t_{0}, p\right)\right)=0$. By the definition of $M$ and Definition 2.7 this implies $\Gamma^{+}\left(t_{0}, \varphi\right) \subset M$. By Lemma 2.3 we have $L^{+}\left(t_{0}, \varphi\right) \subset M$. Since $u\left(t, t_{0}, \varphi\right) \rightarrow L^{+}\left(t_{0}, \varphi\right)$ as $t \rightarrow \infty$ the theorem is proven.

(*) Ann Mat. Pura Appl. (4), 86 (1970), pp. 325.330

(**) Entrata in Redarione il 14 aprile 1971 . 